\title{
Is a Patient With Asymptomatic Esophagitis Really Hyposensitive to Acid and Distention?
}

\author{
Takahisa Yamasaki, Jiro Watari, Tadayuki Oshima, Toshihiko Tomita and Hiroto Miwa* \\ Division of Upper Gastroenterology, Department of Internal Medicine, Hyogo College of Medicine, Nishinomiya, Japan
}

A 62-year-old woman underwent an upper gastrointestinal endoscopy as part of her routine medical check-up in January 2010, when grade B reflux esophagitis was identified (Fig. 1). However, she did not complain any reflux symptoms, and the score by the frequency scale for symptoms of gastroesophageal reflux disease ${ }^{1}$ was 0 , thus she was diagnosed as "asymptomatic esophagitis (AE)". ${ }^{2}$

An acid perfusion test was performed and analyzed according to Fass's method. ${ }^{3}$ Acid perfusion sensitivity score (APSS) was calculated from the duration of typical symptom perception (T) and sensory intensity rating at the end of the acid perfusion (I) is as follows; APSS $=\mathrm{I} \times \mathrm{T} / 100(\mathrm{~cm} \times \mathrm{sec} / 100)$. Her APSS showed zero points because both $\mathrm{T}$ and $\mathrm{I}$ were 0 , which was lower than that of the healthy controls in our hospital (24.2 \pm 24.0$)$.

Next, a Barostat test was performed based on modified Yang's method, ${ }^{4}$ in which the balloon expanded $10 \mathrm{~cm}$ above the lower esophageal sphincter according to the ramp distension protocol (2 mmHg/15 sec) (Fig. 2). Her initial perception threshold which means the minimum value at which the patient first perceive the stimulus was $42 \mathrm{mmHg}$, and the pain threshold, namely the minimum value at which the patient first describe the stimulus as painful, was over $60 \mathrm{mmHg}$, both of which were higher than those of the healthy controls in our hospital $(24.5 \pm 10.4$
$\mathrm{mmHg}$ for initial perception threshold and $41.9 \pm 9.6 \mathrm{mmHg}$ for pain threshold, respectively).

Recent studies on AE from Japan and China showed that prevalence of the disease varied from $12.0 \%$ to $58.8 \%$ of patients with $\mathrm{RE}$ including grade $\mathrm{M}$ (minimal change), $\mathrm{A}$ or $\mathrm{B}$ of reflux

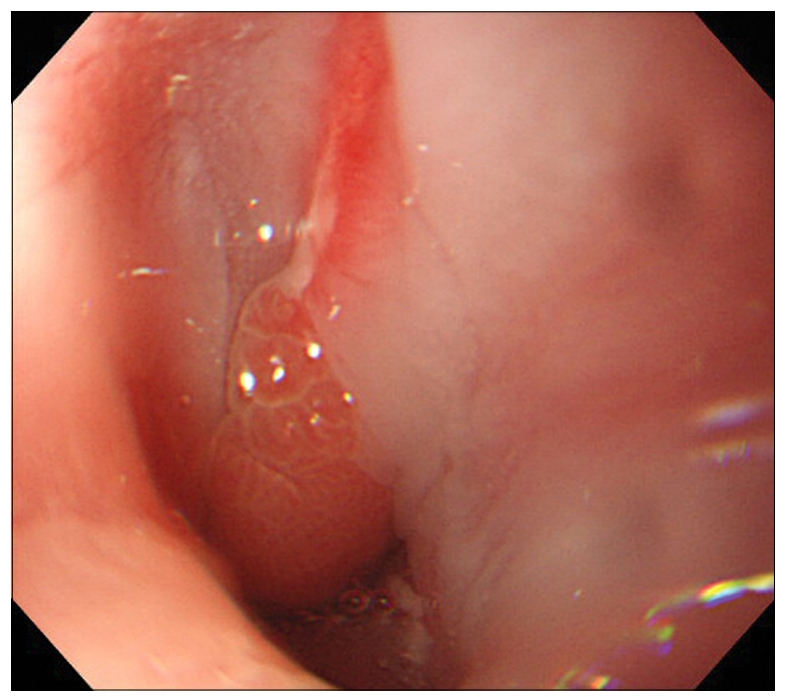

Figure 1. Upper gastrointestinal endoscopic finding shows grade B reflux esophagitis.

Received: May 27, 2011 Revised: June 21, 2011 Accepted: June 22, 2011

(c) This is an Open Access article distributed under the terms of the Creative Commons Attribution Non-Commercial License (http://creativecommons. org/licenses/by-nc/3.0) which permits unrestricted non-commercial use, distribution, and reproduction in any medium, provided the original work is properly cited.

*Correspondence: Hiroto Miwa, MD, PhD

Division of Upper Gastroenterology, Department of Internal Medicine, Hyogo College of Medicine, 1-1 Mukogawa-cho, Nishinomiya, Hyogo 663-8501, Japan

Tel: +81-798-45-6662, Fax: +81-798-45-6661, E-mail: miwahgi@hyo-med.ac.jp

Financial support: None.

Conflicts of interest: None. 


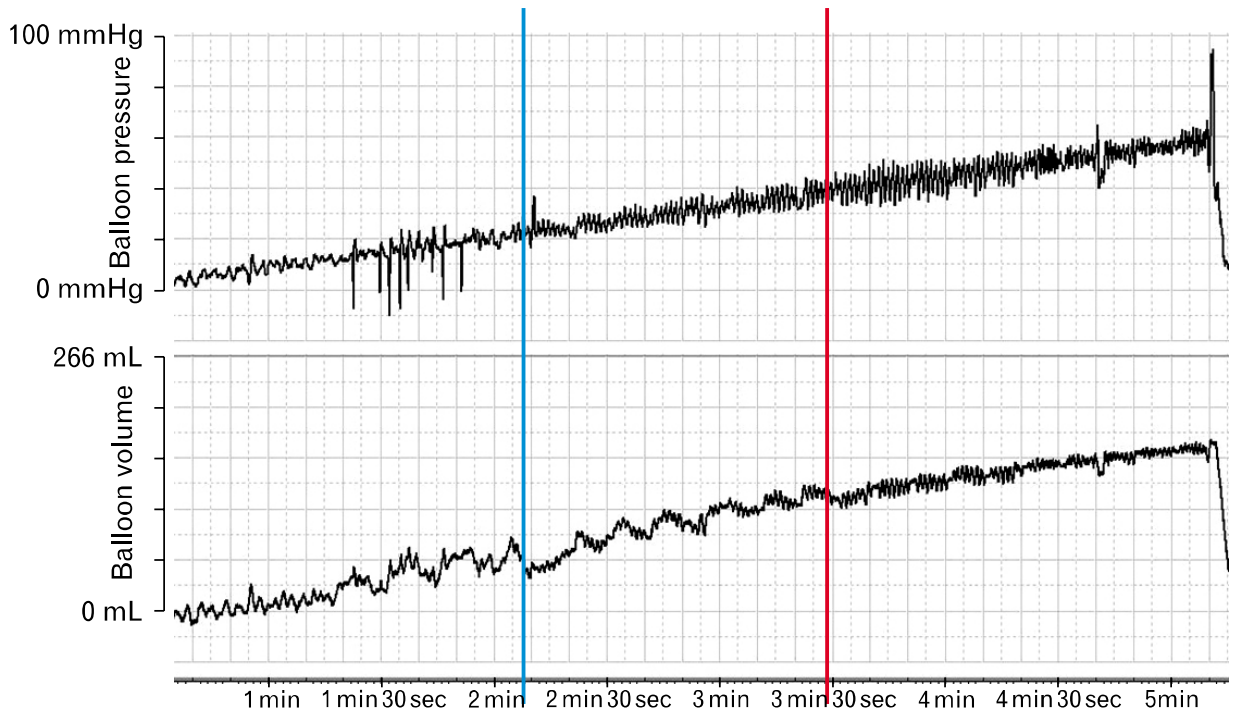

Figure 2. Pressure-volume relationship during fasting isobaric esophageal distensions. The balloon expanded according to the ramp distension protocol (2 $\mathrm{mmHg} / 15 \mathrm{sec})$. The blue and red lines indicate the mean initial perception threshold of healthy controls and the initial perception threshold of this case, respectively. esophagitis. One of the reasons for this variation may depend on the differences in definition of reflux symptoms and the population studied. Although male gender, lower body mass index, drinking and smoking are considered to be significantly and independently associated with $\mathrm{AE}$, its pathogenesis remains controversial. Actually, patients with AE may be hyposensitive to reflux of acid in their esophagus. However, very little is known whether peripheral hyposensitivity, central hyposensitivity or the transient receptor potential vanilloid 1 is involved in the development of AE. ${ }^{5}$ Our case suggests that esophageal hyposensitivity to acid and mechanical stimulus may be associated with the pathophysiology of AE.

\section{References}

1. Kusano M, Shimoyama Y, Sugimoto S, et al. Development and eval- uation of FSSG: frequency scale for the symptoms of GERD. J Gastroenterol 2004;39:888-891.

2. Nozu T, Komiyama H. Clinical characteristics of asymptomatic esophagitis. J Gastroenterol 2008;43:27-31.

3. Fass R, Naliboff BD, Fass SS, et al. The effect of auditory stress on perception of intraesophageal acid in patients with gastroesophageal reflux disease. Gastroenterology 2008;134:696-705.

4. Yang M, Li ZS, Chen DF, et al. Quantitative assessment and characterization of visceral hyperalgesia evoked by esophageal balloon distention and acid perfusion in patients with functional heartburn, nonerosive reflux disease, and erosive esophagitis. Clin J Pain 2010;26:326-331.

5. Miwa H, Kondo T, Oshima T, Fukui H, Tomita T, Watari J. Esophageal sensation and esophageal hypersensitivity - overview from bench to bedside. J Neurogastroenterol Motil 2010;16:353362. 